\title{
Alexander Kluge und die Frühe Neuzeit
}

Alexander Kluge et la première modernité

Alexander Kluge and his relation to early modern culture

\section{Winfried Siebers}

\section{OpenEdition}

\section{Journals}

Édition électronique

URL : http://journals.openedition.org/ceg/1175

DOI : $10.4000 /$ ceg. 1175

ISSN : 2605-8359

\section{Éditeur}

Presses Universitaires de Provence

Édition imprimée

Date de publication : 18 décembre 2015

Pagination : 127-138

ISBN : 979-1-03200-020-5

ISSN : 0751-4239

\section{Référence électronique}

Winfried Siebers, «Alexander Kluge und die Frühe Neuzeit », Cahiers d'Études Germaniques [Online], 69 |

2015, Online erschienen am: 17 Dezember 2017, abgerufen am 27 November 2020. URL : http://

journals.openedition.org/ceg/1175; DOI : https://doi.org/10.4000/ceg.1175 


\title{
Alexander Kluge und die Frühe Neuzeit
}

\author{
Winfried SIEBERS
}

Universität Osnabrück

Wolfgang Adam zum 65. Geburtstag

Das Thema „Alexander Kluge und die Frühe Neuzeit" ist nicht neu - und gleichzeitig ist es für die Betrachtung von Kluges Gesamtwerk höchst evident. Es ist nicht neu, weil in den letzten Jahren verschiedene wissenschaftliche Studien zu diesem Themenbereich erschienen sind, für die sich eine ganze Reihe von Belegen anführen lassen: So hat man z.B. frühneuzeitliche emblematische Strukturen in den Text-Bild-Kombinationen bei Kluge entdecken wollen ${ }^{1}$; man befasste sich mit den polyhistorischen und enzyklopädischen Zügen in Kluges Werk $^{2}$; und schließlich wurde auf die Traditionen des anekdotischen Erzählens und der Kalendergeschichte in den literarischen Arbeiten hingewiesen ${ }^{3}$. Das Thema liegt zudem auf der Hand, denn es ist unmittelbar einsichtig, dass es in Kluges literarischem Werk zahlreiche

1 Vgl. Hyun Soo Cheon, Intermedialität von Text und Bild bei Alexander Kluge. Zur Korrespondenz von Früher Neuzeit und Moderne, Würzburg, Königshausen \& Neumann, 2007; Wilhelm Vosskamp, „Emblematik der Geschichte. Alexander Kluges literarische und filmische Geschichtsschreibung“, in Internationales Archiv für Sozialgeschichte der deutschen Literatur, 36/2, 2011, S. 361-372.

2 Vgl. Rainer Stollmann, „Der unterschätze Mensch. Der Text als anschlussfähiges Netzwerk“, in Hans-Peter Burmeister (Hrsg.), Maßverhältnisse des Politischen. Öffentlichkeit und Erfahrung an der Schwelle zum 21. Jahrhundert. Eine Tagung mit Alexander Kluge und Oskar Negt, Rehburg-Loccum, Evang. Akademie Loccum, 2003, S. 13-24; Winfried Siebers, „Weltkasten mit Digressionen. Spuren der Aufklärung in Oskar Negts und Alexander Kluges gemeinsamer Philosophie“, in Christian Schulte/ Rainer Stollmann (Hrsg.), Der Maulwurf kennt kein System. Beiträge zur gemeinsamen Philosophie von Oskar Negt und Alexander Kluge, Bielefeld, transcript, 2005, S. 201-218; Sven Hanuschek, „Der Blick neben die Diva. Alexander Kluges ,Geschichten vom Kino“ und die Polyhistorie“", in Text + Kritik, Heft 85/86: Alexander Kluge, Neufassung, Gastred. Thomas Combrink, München, Edition Text + Kritik, 2011, S. 39-47; Gunther Martens, „,Wann wird man soweit sein, Bücher wie Kataloge zu schreiben? Alexander Kluge und die enzyklopädische Literatur“, in ibid., S. 128-136.

3 Vgl. Winfried Siebers, „Das Fragezeichen der Poesie. Anekdotisches Erzählen bei Alexander Kluge“, in Klemens Gruber/Christian Schulte (Hrsg.), Die Bauweise von Paradiesen - für Alexander Kluge, Themenheft von Maske und Kothurn, 53/1, 2007, S. 103-111; Wolfgang Reichmann, Der Chronist Alexander Kluge. Poetik und Erzählstrategien, Bielefeld, Aisthesis, 2009; Richard Faber, „Postmodern qua prämodern? Alexander Kluges ,Die Lücke, die der Teufel läßt‘ im Vergleich mit Johann Peter Hebel und anderen Erzählern des 19. Jahrhunderts“, in Simone Schröder et al. (Hrsg.), Odysseus - Passagiere. Über Selbstbestimmung und Determination in Literatur, Medien und Alltag, Würzburg, Königshausen \& Neumann, 2011, S. 55-72; Joseph Vogl, „Kommentar zu ,Die Lücke, die der Teufel läßt““, in Text + Kritik, Heft 85/86, S. 120-124. 
Anknüpfungspunkte an die Epoche der Frühen Neuzeit gibt. So greift er etwa wie eben erwähnt - in seinen erzählerischen Arbeiten auf beliebte Gattungsformen jener Epoche wie die Anekdote, die Kalendergeschichte oder den Dialog zurück. Oder es gibt direkte inhaltliche Bezüge, wie sie sich am Beispiel der ausführlichen Auseinandersetzung mit dem 1678 erschienenen Roman La Princesse de Clèves der Madame de La Fayette in der Chronik der Gefühle (2000) und im Labyrinth der zärtlichen Kraft (2009) zeigen lassen. Schließlich hat sich Kluge in programmatischen Reden, Interviews und Stellungnahmen häufiger selbst zu seinem allgemeinen Verhältnis zu dieser Epoche sowie zu den eigenen inhaltlichen und personalen Bezugspunkten innerhalb der Epoche geäußert.

Verweise auf die Frühe Neuzeit sind aber auch in den Fernseharbeiten Kluges zu finden, vor allem in den Interviewsendungen, in denen immer wieder auf Ereignisse, Personen oder Denkmotive aus dem 16., 17. oder 18. Jahrhundert eingegangen wird. Bei einer wenngleich nur groben Durchsicht der beiden vorliegenden Titelbibliographien für die Fernsehkulturmagazine aus dem Zeitraum 1988 bis 2007 ergibt sich eine Anzahl von knapp 80 Magazinen, die eindeutig Stoffe, Motive, Themen oder Personen der Frühen Neuzeit behandeln. ${ }^{4}$ Das Problem dieser beiden Verzeichnisse ist allerdings ihre Knappheit - denn nicht immer ist der thematische Kern einer entsprechenden Sendung aus dem Ober- und Untertitel der mitunter sehr verschlüsselten Formulierungen ablesbar. Eine Zuordnung ist deshalb oft schwierig, so dass die Zahl der auf die Frühe Neuzeit bezogenen Magazine wesentlich höher ausfallen würde, wenn man eine inhaltsanalytische Erschließung der Fernsehmagazine vornehmen könnte.

Die Epochenbezeichnung „Frühe Neuzeit“" ist bisher ohne weitere Erläuterung eingeführt worden. Nach einer inzwischen über dreißigjährigen Begriffsgeschichte ist der Terminus zwar „fest etabliert“" , als abkürzende Verständigungskategorie jedoch oft im Bereich der auf diese Epoche spezialisierten Fachvertreter der verschiedensten Disziplinen verblieben. Es ist deshalb ratsam, an dieser Stelle eine kurze Bemerkung zur zeitlichen Abgrenzung der Epoche einzufügen: Die Frühe Neuzeit ist nach allgemeinem Verständnis durch die historischen Eckdaten 1500 und 1800 gekennzeichnet - der Zeitraum kann in der knappen Formel ,von der Reformation zur (Französischen) Revolution' zusammengefasst werden. Dabei stehen die beiden genannten Daten als Chiffren für vielfältige Ereigniskomplexe, die sich um 1500 (u.a. ,Entdeckung Amerikas 1492, Reichsreform 1495, Luthers Thesenanschlag 1517) und um 1800 (u.a. Französische Revolution seit 1789, Auflösung des Alten Reiches 1806, Wiener Kongress 1814/15) bündeln. ${ }^{6}$ Literatur- und Kulturhistoriker des deutschsprachigen Raums würden eine leicht abweichende Definition geben - für sie steht eher die Durchsetzung des Buchdrucks seit 1450 und die Ablösung der rhetorisch dominierten

4 Vgl. Christian Schulte, „Kulturmagazine 1988-1999“, in Christian Schulte (Hrsg.), Die Schrift an der Wand. Alexander Kluge - Rohstoffe und Materialien, Osnabrück, Universitätsverlag Rasch, 2000, S. 401-438; Beata Wiggen, „Kulturmagazine 1999-2007“, in Klemens Gruber/Christian Schulte (Hrsg.), Die Bauweise von Paradiesen, S. 137-192.

5 Herbert Jaumann, „Frühe Neuzeit“, in Klaus Weimar (Hrsg.), Reallexikon der deutschen Literaturwissenschaft, Bd. 1, Berlin/New York, de Gruyter, 1997, S. 632-636, hier S. 633.

6 Vgl. Karl Vocelka, Frühe Neuzeit 1500-1800, Konstanz/München, UVK, 2013, S. 11f. 
Kulturproduktion durch neu aufkommende Konzepte der Autonomieästhetik um 1750 im Vordergrund. In komparatistischer Perspektive kann zudem ,für eine europäische Makroepoche“ plädiert werden, „die aus literaturwissenschaftlicher Sicht vom späten 14. bis zum frühen 18. Jahrhundert reicht". ${ }^{7}$ Die Frühe Neuzeit als Makroepoche umfasst in diesem Sinne die Teilepochen Renaissance, Reformationszeit, Späthumanismus, ,Barock‘ und Aufklärung. Die Einheit dieser Epoche, die gelegentlich als „Inkubationszeit der Moderne“ (Paul Münch) oder als „Musterbuch der Moderne“ (Winfried Schulze) bezeichnet wurde, ist jedoch unbestritten. ${ }^{8}$

Das Thema „Kluge und die Frühe Neuzeit“ soll unter drei Aspekten behandelt werden. In einem ersten Teil werden die oft nur punktuellen programmatischen Bezüge Kluges auf die Frühe Neuzeit vorgestellt. In einem zweiten Abschnitt wird sehr knapp an nur einem Beispiel eine inhaltliche Bezugnahme Kluges auf die Epoche anhand des Romans Die Prinzessin von Clèves der Madame de La Fayette aus dem Jahre 1678 skizziert. In einem dritten und letzten Teil wird zu zeigen versucht, dass Kluges Umgang mit der Geschichte, seine Gebrauchsweise der Geschichte - die einen ausgesprochenen Sammlungscharakter trägt und eine immense Themenbreite zeigt - mit Organisationsformen des Wissens in der Frühen Neuzeit in Verbindung $\mathrm{zu}$ bringen ist.

\section{I.}

In Preisreden, Interviews und anderen Stellungnahmen hat Kluge hin und wieder Auskunft über sein Verhältnis zur literarischen Tradition der Frühen Neuzeit gegeben. Diese Bezugnahmen sind oft nur punktuell, beiläufig und wenig systematisch geäußert worden, doch lassen sich ganz deutlich zwei Denkströmungen bzw. frühneuzeitliche Teilepochen als Referenzpunkte angeben, die nach Kluges Auffassung auch für die Gegenwart noch Problemlösungen bereithalten: es sind dies der Humanismus und die Aufklärung. Beide Bezugsepochen stehen unter Kluges Leitsatz „Erfahrungen noch einmal prüfen“9 , das heißt: aus den überlieferten Erfahrungszusammenhängen historischer Konflikte, die in der Literatur und der Geschichte vergangener Epochen bereitliegen, Lösungsmöglichkeiten für den Umgang mit ähnlichen Problemlagen

7 Wolfgang Adam, „Frühneuzeit-Forschung in der Zeitschrift ,Euphorion““, in Euphorion, 101, 2007, S. 451-493, hier S. 453; vgl. auch Wolfgang Adam, „Bibliotheksgeschichte und FrühneuzeitForschung. Bilanz und Perspektiven am Beispiel des Nachlaßverzeichnisses von Fürst Ludwig von Anhalt-Köthen", in Euphorion, 102, 2008, S. 1-38; vgl. die neueren Studienbücher: Kai Bremer, Literatur der Frühen Neuzeit. Reformation - Späthumanismus - Barock, Paderborn, Fink, 2008; Andreas Keller, Frühe Neuzeit. Das rhetorische Zeitalter, Berlin, Akademie-Verlag, 2008; Iwan-Michelangelo D’Aprile/ Winfried Siebers, Das 18. Jahrhundert. Zeitalter der Aufklärung, Berlin, Akademie Verlag, 2008.

8 Paul Münch, „Einleitung“, in Paul Münch (Hrsg.), Ordnung, Fleiß und Sparsamkeit. Texte und Dokumente zur Entstehung bürgerlicher Tugenden, München, Dt. Taschenbuch-Verlag, 1984, S. 9-38, hier S. 15; Winfried Schulze, „,Von den großen Anfängen des neuen Welttheaters‘. Entwicklung, neue Ansätze und Aufgaben der Frühneuzeitforschung", in Geschichte in Wissenschaft und Unterricht, 44/12, 1993, S. 3-18, hier S. 8.

9 Alexander Kluge, Die Kunst, Unterschiede zu machen, Frankfurt am Main, Suhrkamp, 2003, S. 27. 
der Gegenwart abzuleiten. Ganz in diesem Sinne schlägt Kluge in der Rede zur Entgegennahme des Bremer Literaturpreises im Jahre 2001 folgendes vor:

\begin{abstract}
Alle Impulse der Aufklärung, je früher sie angesetzt waren, sollten noch einmal aufgenommen werden. Die Aufklärung entstand eigentlich im 17. Jahrhundert. Hundert Jahre vor der Französischen Revolution finden wir in Frankreich und in Deutschland [...] eine Aufklärung, die [...] die subjektive Seite des Menschen einbezieht. [...] An diese Form der Aufklärung unser 21. Jahrhundert anzukoppeln und eine Eröffnungsbilanz zum 21. Jahrhundert neu zu schreiben und alle Gefühle, auch die Irrtümer, [...] noch einmal abzuklopfen und auf den Prüfstand zu stellen, das ist etwas, was uns zu Beginn des 21. Jahrhunderts [...] gut ansteht. Dies sollte geschehen unter Anknüpfung an Montaigne, an alle diese Philosophen, Projektemacher, Dichter, die nach den blutigen Bürgerkriegen und Religionskriegen, welche die frühe Neuzeit kennzeichnen (die Hugenottenkriege in Frankreich, der Dreißigjährige Krieg bei uns), einen Neuanfang setzten. ${ }^{10}$
\end{abstract}

Und weiter, in einer Äußerung aus dem Jahre 2003: „Im 16. und im 17. Jahrhundert liegen starke Spuren einer Aufklärung, die sich im Zwerchfell festmacht, in der Beharrlichkeit. Wenn die Aufklärung des 18. Jahrhunderts nicht ausreichte, um den Faschismus zu besiegen, muß man weitergraben und nach den früheren Stationen der Aufklärung fragen“. ${ }^{11}$

Man sieht, dass die Leitfrage von einem Gegenwartsinteresse herrührt, nämlich der konfliktreichen kriegerischen Realität des 20. und 21. Jahrhunderts, und dass die Suche nach einem Neuanfang mit Hilfe der historisch bereits gemachten Erfahrungen ins Werk gesetzt werden soll. Hierzu werden die Friedensutopien des europäischen Humanismus und die philosophischen Impulse der Aufklärung in Erinnerung gerufen. Diese Erinnerungsarbeit ist auch ein Hauptanliegen in Kluges literarischen Arbeiten. So heißt es etwa in der Schiller-Preisrede von 2003: „Ich empfinde den starken Wunsch, unser 21. Jahrhundert, als Waggon betrachtet, an dieses 18. Jahrhundert anzukoppeln und nicht an die Giftbecher des 19. und 20. Jahrhunderts. Das ist das Pathos und der zugrundeliegende rote Faden in Chronik der Gefühle ". ${ }^{2}$

Humanismus und Aufklärung sind auch deshalb herausgehobene Epochen von Kluges intellektueller Orientierung, weil sie einerseits den Zugang zur Antike eröffnen - er nennt sie einmal „Relaisstationen zur Antike“"13. Andererseits ist über beide Epochen eine Verbindung zur Kritischen Theorie herstellbar. In einem Interview aus dem Jahr 2004 gefragt, in welcher literarischen Tradition er sich sähe, antwortet Kluge: ,[...] in eine Tradition stellen, da würde ich etwas ganz Einfaches nehmen: Ovid, Tacitus, Montaigne“14. Und wenig später äußert er in demselben Interview auf die Frage nach seiner literarischen „Abstammung“:

${ }^{10}$ Alexander Kluge, „Der ,Quotenkiller“ und die Sprache der Aufklärung. Rede zum Bremer Literaturpreis 2001“, in Alexander Kluge, Personen und Reden, Berlin, Wagenbach, 2012, S. 61-66, hier S. 66.

11 Kluge, Die Kunst, Unterschiede zu machen, S. 27.

12 Alexander Kluge, „Einen Moment lang schien die Gesellschaft wie ein Garten: Silvester 1799. Rede zum Schiller-Gedächtnispreis 2001“, in Kluge, Personen und Reden, S. 52-60, hier S. 54.

13 Alexander Kluge/Thomas Combrink, „,Wie erkennt man einen Dämon? Er schwatzt und übertreibt.' Gespräch mit Alexander Kluge“, in Neue deutsche Literatur, 52/560, 2004, S. 47-56, hier S. 54.

14 Ibid. 
Ich stamme von der Kritischen Theorie, und die haben eine ganz feste Genealogie. Horkheimer hat das frühe Bürgertum, die Menschen um 1600, also Shakespeare, Galilei, Monteverdi, den Homo novus, als Ahnvater, und Montaigne ist sozusagen genau dieser Typus. Montaigne ist mein Vorbild, denn er weist direkt auf die Antike. ${ }^{15}$

Schließlich wird Kluge zu denjenigen Romanautorinnen und -autoren befragt, die ihm wichtig sind. Er nennt Tolstoi, Flaubert, Fontane, selbstverständlich Ovid, doch die zentrale Äußerung in dieser Passage mag überraschen: „Mein Lieblingsroman ist die ,Princesse de Clèves“ von Madame de La Fayette“" 16.

Aus dem bisher Gesagten kann das folgende Zwischenresümee gezogen werden:

1. Die Epoche der Frühen Neuzeit ist für Kluge eine „Relaisstation“ zwischen Vergangenheit und Gegenwart, die Verknüpfungen zur Antike ebenso zulässt wie zur Kritischen Theorie.

2. Die historische Signatur der Frühen Neuzeit mit ihren konfessionellen Auseinandersetzungen und europäischen Kriegen erlaubt - so Kluge - die Wiederaufnahme unerledigter Fragestellungen dieser Epoche für die Gegenwart. Die Erfahrungsgehalte der Vormoderne können für die Moderne des 20. und 21. Jahrhunderts fruchtbar gemacht werden.

3. Charakteristisch für Kluges historisches Verfahren ist die Suche nach Anfangsund Ursprungssituationen, in denen bestimmte Problemlagen oder Diskursfelder sich entwickeln. Mit Montaigne und Madame de La Fayette sind zwei Diskursbegründer benannt, die für Kluges Schreibweise wichtig sind: Montaigne begründet die Gattung des Essays, in dem ein Thema im Wechselspiel von Sachlichkeit und Subjektivität in einem offenen Denkprozess dargelegt wird. Madame de La Fayette begründet den psychologischen Roman in dem Sinne, dass hier der Konflikt von Leidenschaft und Vernunft erstmals in quasi dokumentarischer Form nachgezeichnet wird.

\section{II.}

Allein schon der Umfang und die Mehrfachverwendung in unterschiedlichen Kontexten hebt Kluges Beschäftigung mit seinem „Lieblingsroman“ aus der Zahl positiver Bezugnahmen auf die Frühe Neuzeit heraus. Erstmals erwähnt wird er bereits 1965 im Zusammenhang einer Diskussion, bei der es um die Funktion von Typisierung und Differenzierung in der Literatur und im Film geht. ${ }^{17}$ Ausführlicher wird dem Leser der Chronik der Gefühle im Jahr 2000 eine fünfseitige Text-BildMontage als „Kommentar“ zur Prinzessin von Clèves präsentiert, in der wesentliche Einzelelemente des Romans erläutert und in Zusammenhang mit Niklas Luhmanns Studie Liebe als Passion (1982) gebracht werden. ${ }^{18}$ Beträchtlich auf rund 40 Seiten

15 Ibid.

${ }^{16}$ Ibid., S. 55.

${ }_{17}$ Vgl. Edgar Reitz/Alexander Kluge/Wilfried Reinke, „Wort und Film“, in Klaus Eder/Alexander Kluge, Ulmer Dramaturgien. Reibungsverluste. Stichwort: Bestandsaufnahme, München, Hanser, 1980, S. 9-27, hier S. 17, Erstdruck in Sprache im technischen Zeitalter, 13, 1965, S. 1015-1030.

18 Vgl. Alexander Kluge, Chronik der Gefühle, 2 Bde., Frankfurt am Main, Suhrkamp, 2000, Bd. 1, S. 939-943. 
erweitert und um einen Geschichtenzyklus zu Luhmanns Buch ergänzt, bildet der Kommentar dann knapp zehn Jahre später ein eigenes Kapitel im Labyrinth der zärtlichen Kraft (2009). ${ }^{19}$ Diese Version wird fast wörtlich zunächst als Nachwort für eine Neuausgabe des Romans im renommierten Manesse-Verlag übernommen (2011) sowie nahezu unverändert erneut in Das fünfte Buch (2012) abgedruckt. ${ }^{20}$

Der historische Hintergrund des 1678 zunächst anonym erschienenen Romans La Princesse de Clèves der Madame de La Fayette (Marie-Madeleine Pioche de la Vergne, comtesse de La Fayette, 1634-1693) ist die Darstellung des französischen Hofes in der Regierungszeit Heinrichs II. in den Jahren 1547 bis 1559. Erzählt wird von der sehr jungen und unerfahrenen, aber außerordentlich schönen Hofdame von Chartres, die auf Anraten ihrer Mutter den ehrenwerten, aber ungeliebten Fürsten von Clèves heiratet. Kurz nach der Heirat kommt die nunmehrige Prinzessin von Clèves in Kontakt mit dem attraktiven Herzog von Nemours, in den sie sich leidenschaftlich verliebt. Um nicht in ein höfisches Spiel von Verbergung, Heimlichkeiten und Klatsch hineingezogen zu werden, gesteht die Prinzessin ihrem Gatten die bisher nur auf tugendhafte Weise gelebte Liebe zu dem Herzog. Der Fürst von Clèves stirbt kurz darauf, innerlich gebrochen. Obwohl sie nun frei wäre für ein Leben mit dem Herzog von Nemours, verzichtet die Prinzessin auf die Fortsetzung der Beziehung und zieht sich aus der höfischen Welt in ein Kloster zurück. ${ }^{21}$

Kluge legt in seinem Kommentar zunächst die zentralen Erzählmotive des Romans dar. Sodann erläutert er einige der charakteristischen Szenen, die er in den Bezugsrahmen des zeitgenössischen Liebesdiskurses und eines neuen Menschenbildes rückt, das vom Neostoizismus geprägt ist. Dieser ordnet die Gefühle dem Willen und der Vernunft unter, er propagiert emotionale Selbstbeherrschung, Gelassenheit und Seelenruhe in den Wechselfällen des Lebens. Schließlich stellt Kluge unter Hinweis auf die Studie Liebe als Passion des Soziologen Niklas Luhmann einige „moderne Fragen“ an den Roman, die dessen Aktualität für die Gegenwart aufschließen sollen. Dabei verfährt er nach der bereits dargestellten Methode der Fortschreibung historisch aufgeworfener Fragestellungen für die Gegenwart. So heißt es über den Roman aus dem 17. Jahrhundert:

Die Landkarte der Gefühle, welche Madame de La Fayette für ihren Roman voraussetzt, gestattet nicht nur den Vergleich mit anderen Romanen, sondern auch den mit Erfahrungen des 20. und 21. Jahrhunderts, ja mit solchen in den USA, Kanada, Australien oder in Asien, die keine plausible Verknüpfung mit den Geschehnissen um die Prinzessin von Clèves und den Herzog von Nemours besitzen. Vergleichen heißt: Unterschiede machen.

19 Vgl. Alexander Kluge, Das Labyrinth der zärtlichen Kraft. 166 Liebesgeschichten, Frankfurt am Main, Suhrkamp, 2009, S. 443-517.

${ }^{20}$ Vgl. Alexander Kluge, „Nachwort“, in Madame de La Fayette, Die Prinzessin von Clèves, aus dem Franz. übers. von Ferdinand Hardekopf, überarb. und komm. Neuausgabe, Zürich, Manesse, 2011, S. 347-364; Alexander Kluge, Das fünfte Buch. Neue Lebensläufe. 402 Geschichten, Berlin, Suhrkamp, 2012, S. 277-304.

${ }^{21}$ Zur Rezeption des Romans im deutschsprachigen Raum mit Hinweisen zur Deutungsgeschichte vgl. Andrea Grewe, „Où sont les dames d'antan. Erinnerungslücken im literarischen Gedächtnis. Das Werk Marie-Madeleine de Lafayettes im deutschen Sprachraum“, in Jan Standke unter Mitwirkung von Holger Dainat (Hrsg.), Gebundene Zeit. Zeitlichkeit in Literatur, Philologie und Wissenschaftsgeschichte. Festschrift für Wolfgang Adam, Heidelberg, Winter, 2014, S. 529-541. 
Nichts in dem frühmodern-antiken Roman entspricht unmittelbar dem inzwischen durch Mutation und Selektion veränderten ,System ‘ der Liebe. ${ }^{22}$

Schließlich kommt er zu der Frage: „Wie würde man heute den Roman Die Prinzessin von Clèves weiterschreiben? Wäre das im 21. Jahrhundert möglich?“. ${ }^{23}$ Im Rückgriff auf ein Interview des Romanisten Anselm Haverkamp versteht Kluge die „Fortschreibung der Prinzessin von Clèves“ als „operativ und offensiv“, Kompromisse seien zu vermeiden, die Maximalwerte für die Ideale der „Einmaligkeit, Selbstachtung, Authentizität, Befähigung zur [...] Treue“ dürften nicht unterschritten, das „Schicksal des Liebesideals in der Massengesellschaft“ müsse von geeigneten Literaten angemessen behandelt werden. Dabei könnten Autoren der literarischen Moderne wie Joyce, Döblin, Dos Passos oder Steinbeck behilflich sein. ${ }^{24}$

Die höchst differenzierte Perspektive Kluges im Umgang mit dem Roman aus dem 17. Jahrhundert kann hier nur angedeutet werden. Kluges Kommentar ist kulturgeschichtlich zutreffend und klar strukturiert - er beschreibt die zugrunde liegende Konfliktkonstellation in ihren historischen Entstehungsbedingungen und aktuellen Bezügen. Es ist kein Wunder, dass der Text ohne Abstriche als Nachwort in einer vor zwei Jahren erschienenen Neuausgabe des Romans übernommen wurde - denn er enthält alles, was ein Leser zur Erschließung der Romanvorlage benötigt. Die Fassung im Labyrinth der zärtlichen Kraft ist jedoch zusätzlich von Text-BildMontagen begleitet, die den Romankommentar auf einer Art zweiten Ebene in einen dialogischen Prozess einbinden. ${ }^{25}$

Auch aus dieser beispielhaften, hier nur kurz skizzierten inhaltlichen Bezugnahme Kluges auf einen Text der Frühen Neuzeit möchte ich zwei Beobachtungen festhalten:

1. Wieder begegnet hier die Suche nach einer Anfangs- oder Ursprungssituation, in der eine Problemlage der Lebenserfahrung in einem spezifischen historischen Kontext erstmals künstlerisch anspruchsvoll aufgearbeitet wird. Kluge formuliert das zusammenfassend mit den folgenden Worten: „Die Prinzessin von Clèves ist der erste Roman praktischer Aufklärung, eine Fibel der praktischen Urteilskraft auf dem riskantesten Gebiet der Lebenserfahrung, dem Gebiet der ZÄRTLICHEN KRAFT". ${ }^{26}$

2. Und ebenfalls zeichnet sich hier die bereits erwähnte charakteristische Denkfigur Kluges ab, dass Erfahrungsgehalte vergangener Epochen für die Deutung und Kommentierung aktueller Gegenwartsfragen bedeutsam sind, und sei es „nur“ darin, Differenzen erkennen zu können.

${ }^{22}$ Kluge, Das Labyrinth der zärtlichen Kraft, S. 455.

23 Ibid., S. 465.

24 Ibid.

${ }^{25}$ Diese intermediale Verweisstruktur muss hier nicht eigens erörtert werden, da sie von Nicoletta Wojtera sehr detailliert und völlig überzeugend dargelegt wurde; vgl. Nicoletta Wojtera, „Lesen und Verwandlung als intermediale Relation", in Steffen Groscurth/Thomas Ulrich (Hrsg.), Lesen und Verwandlung. Lektüreprozesse und Transformationsdynamiken in der erzählenden Literatur, Berlin, Frank \& Timme, 2011, S. 43-54.

${ }^{26}$ Kluge, Das Labyrinth der zärtlichen Kraft, S. 445. 


\section{III.}

Neben den programmatischen Stellungnahmen und den inhaltlichen Bezugnahmen gibt es eine dritte Ebene der Rezeption der Frühen Neuzeit bei Kluge, denn bei der Präsentation verschiedener Werksegmente bedient er sich verschiedener wissenssammelnder und wissensorganisierender Verfahren aus der Frühen Neuzeit. Am offensichtlichsten ist das bei dem Enzyklopädiegedanken, wobei Kluge hier fast immer gezielt an die große Encyclopédie der französischen Aufklärung anknüpft, die von Jean-Baptiste le Rond d'Alembert und Denis Diderot herausgegeben wurde. ${ }^{27}$

Bereits 1981 wird in Geschichte und Eigensinn die Fortschreibung eines solchen Unternehmens ins Auge gefasst, denn es sei „eine einfache Arbeit, Leute zu fragen, d. h. weltweit die selbstversorgende Intelligenz- und Erfahrungspraxis zu versammeln; und es wäre vorstellbar, daraus Enzyklopädien von außerordentlicher Aktualität zu gestalten. Man müsste nur hingehen und fragen" ${ }^{28}$. Aufgenommen wird der Gedanke in einem Fernsehgespräch mit Oskar Negt zum „Projekt: Enzyklopädie“" 29 , wo ein Bogen von dem französischen Grundwerk Diderots bis zu einer „Enzyklopädie des 20. Jahrhunderts“ geschlagen wird, die noch zu schreiben wäre. Fortgeführt werden die Überlegungen anlässlich einer mehrtägigen Veranstaltung zur Präsentation von Der unterschätzte Mensch im Januar 2002. Die Idee der Enzyklopädie, so Kluge, bedürfe einer „Formänderung“, die „,mehrsprachig“ sein müsse in dem Sinne, dass sie für „alle Gesellschaftsschichten“ verstehbar sei. Es gehe darum, „Stück für Stück Unterscheidungsvermögen auf[zu]sammeln“ und mit einer derartigen „Sammelaktion Enzyklopädie“ eine „Wiederaufnahme der Moderne“ und eine „Reinschrift der Aufklärung unter Einbeziehung der Dialektik der Aufklärung“" zu betreiben. ${ }^{30}$

Angesichts von rund 3800 Fernsehmagazinsendungen, darunter ein beträchtlicher Teil an Interviews mit Experten aller Art, könnte man sagen, dass diese Kluge'sche Enzyklopädie des 20. und 21. Jahrhunderts längst existiert. Dabei sollte man sich aber von der Vorstellung eines lexikonartig zusammengestellten systematischen Inventars lösen. Vielmehr geht es - darauf hat Christian Schulte zuletzt noch einmal hingewiesen - um eine „Enzyklopädie der Erfahrung“31, die erst in zweiter Linie um die Einsammlung von Resultaten etwa aus der Nanotechnologie, Biomedizin, Hirnforschung, Kriminologie, Wirtschaftstheorie, Geschichtswissenschaft oder Musik- und Filmhistorie bemüht ist. Demgegenüber seien für Kluge zumeist die „darunterliegenden Motivschichten und individuellen Antriebe“ wichtiger,

${ }^{27}$ Das Folgende ist eine Teilzusammenfassung von Siebers, „Weltkasten mit Digressionen“, S. 201-218.

${ }^{28}$ Alexander Kluge/Oskar Negt, Der unterschätzte Mensch. Gemeinsame Philosophie in zwei Bänden, Frankfurt am Main, Zweitausendeins, 2001, Bd. 2, S. $443 \mathrm{f}$.

${ }^{29}$ Vgl. Alexander Kluge/Oskar Negt, „Projekt: Enzyklopädie/Gebrauchswert von Wissen im 20. Jahrhundert“, 10 vor 11, RTL, 23.07.1990; gedruckt in Negt/Kluge, Der unterschätzte Mensch, Bd. 1, S. $69-80$.

${ }^{30}$ Gesprächsbeitrag Kluges in Burmeister (Hrsg.), Maßverhältnisse des Politischen, S. 158f.

${ }^{31}$ Christian Schulte, „Enzyklopädie der Erfahrung“, in Christian Schulte (Hrsg.), Die Frage des Zusammenhangs. Alexander Kluge im Kontext, Berlin, Vorwerk 8, 2012, S. 9-12, hier S. 11f. 
die $\mathrm{zu}$ diesen Resultaten führen, da den wissenschaftlichen Erkenntnissen oft lebensgeschichtliche „Initialmomente“ zugrunde lägen. ${ }^{32}$

Neuerdings ist - u.a. von Gunther Martens - verstärkt darauf aufmerksam gemacht worden, dass das enzyklopädische Prinzip auch in den literarischen Arbeiten Kluges selbst erkennbar ist. In der Kapiteleinteilung, in der immensen Themenbreite, in der Kürze der Geschichten, in der Verwendung von Fußnoten und Registern, in den Verfahren der Textanordnung mit „markierten Querverweisen“ und dem Gebrauch von Abbildungen lassen sich Elemente einer enzyklopädischen Erzählliteratur finden, die wissensorganisatorisch auf die Literatur der „Barockzeit“, auf die „Tradition der Gelehrsamkeit und Polyhistorie“ zurückzuführen ist. ${ }^{33}$ Am Beispiel der Geschichten vom Kino (2007) hat Sven Hanuschek diesen polyhistorischen Blick Kluges nachverfolgt: Der anti-hierarchische Zug des Polyhistorismus, dem es zunächst um die ungeteilte Inventarisierung des zugänglichen Wissens geht, erlaubt es dem Erzähler der Geschichten vom Kino, zwar auch von prominenten Gestalten der Filmgeschichte (Schauspielern, Regisseuren, Produzenten) zu berichten, genauso wichtig sind ihm aber überdies die heute vergessenen Protagonisten, Kleindarsteller, Gescheiterten oder Nebenfiguren der Kinogeschichte. ${ }^{34}$ Der Polyhistorismus, die „Vielwisserei“, wie man den Terminus übersetzten könnte, ist sozusagen die „ungeordnete Seite des enzyklopädischen Zeitalters“35. Er ist durch einen universalen Wissensbegriff gekennzeichnet, der auf der Methode der Kompilation beruht und prinzipiell anti-systematisch angelegt ist. Dabei wurde die „bunte“ Aufreihung und Zusammenstellung des Wissens sogar als eigene ästhetische Qualität propagiert, die Flexibilität und Beweglichkeit garantierte und die den elaborierten Ordnungsmodellen der Zeit bewusst entgegengestellt wurde. ${ }^{36}$ Allerdings ist diese Art der Wissensorganisation seit Mitte des 17. Jahrhunderts zunehmend kritisiert worden, da die schiere Menge an Beobachtungsdaten und das Problem ihrer wissenschaftstheoretischen Durchdringung mit diesem Verfahren nicht mehr zu bewältigen war.

An dieser Stelle wäre zu fragen: Besteht tatsächlich eine Verbindung zwischen dieser Art des frühneuzeitlichen Polyhistorismus und den Präsentationsformen gegenwärtigen Wissens in den unterschiedlichen Segmenten der literarischen, theoretischen und medialen Arbeiten Kluges? Eine Antwort auf diese Frage kann hier abschließend nur angedeutet werden. Tatsächlich gibt es ein Modell der Wissenspräsentation in der Frühen Neuzeit, dass erstaunliche Parallelen zur „Wissensordnung“ - wie man sagen könnte - von Kluges ästhetischer und medialer Arbeit aufweist. Es handelt sich dabei um die populären Wissenssammlungen, die neben den gelehrten Zeitschriften am Ende des 17. Jahrhunderts eine große Verbreitung fanden.

32 Ibid., S. 11.

${ }^{33}$ Vgl. Martens, ,,Wann wird man soweit sein, Bücher wie Kataloge zu schreiben?““, S. 130.

${ }^{34}$ Vgl. Hanuschek, „Der Blick neben die Diva“, z.B. S. 43.

${ }^{35}$ Flemming Schock, „Wissensliteratur und ,Buntschriftstellerei ${ }^{6}$ in der Frühen Neuzeit: Unordnung, Zeitkürzung, Konversation. Einführung“, in Flemming Schock (Hrsg.), Polyhistorismus und Buntschriftstellerei. Populäre Wissensformen und Wissenskultur in der Frühen Neuzeit, Berlin/Boston, de Gruyter, 2012, S. 1-20, hier S. 3.

${ }^{36}$ Ibid., S. 5. 
So erschien von 1681 bis 1691 das Wochenblatt Denckwürdigkeiten der Welt Oder... Relationes Curiosae, dass der Hamburger Romanautor und Polyhistor Eberhard Werner Happel (1647-1690) herausgab und das als erste deutschsprachige Zeitschrift bezeichnet werden kann. ${ }^{37}$ In dem Blatt kann man Auszüge aus Reisebeschreibungen, politische und historische Berichte, Nachrichten von Bibliotheken und Naturphänomenen, Anekdoten, Geschichten und seltsame Begebenheiten von Tieren und Menschen ebenso finden wie die Präsentation wissenschaftlicher Experimente, Gespenster-, Teufels- und Wundergeschichten sowie Anleitungen zu mathematischen Rechenoperationen. Dies alles wird in den unterschiedlichsten Erzähl- und Darstellungsformen ausgebreitet, allerdings nicht systematisch, sondern in zufälliger Reihung und ohne eine sichtbare Kategorisierung. Selbstverständlich ist die Zeitschrift reich illustriert, die Text-Bild-Relationen sind sorgfältig komponiert und bieten Anlass für die stoffliche Kontrastierung des vielgestaltigen Materials.

Er versuche - so der Redakteur Happel - „die angenehmste Ordnung in einer stets veränderlichen Unordnung“ ${ }^{38}$ darzustellen. Dies ist als eine „Ästhetik chaotischen Wissens" bezeichnet worden, die vom Blattmacher bewusst reflektiert worden sei. Die Nicht-Ordnung resultiere auch aus einer zunehmenden zivilisatorisch bedingten Zeitnot, so dass die Erzählsegmente so gestaltet seien, dass sie innerhalb einer Viertelstunde zu lesen seien und zu einem anderen Zeitpunkt an anderer Stelle wieder mit der Lektüre eingesetzt werden könne. Der Redakteur betont, dass dem Leser eine „[...] angenehme Confusion und Unordnung ohne Zweiffel“ besser gefiele ,als eine nette Ordnung/welche [zwar] leicht zu erfinden“, aber sehr schwer durchzuhalten sei. ${ }^{39}$ Ohne alle Parallelen hier im Einzelnen auszuführen, so wird jeder Rezipient von Kluges Werk einzelne Elemente wiedererkannt haben: die immense Themenbreite, das Nebeneinander von Fakten und Fiktionen, die Anverwandlung naturwissenschaftlicher Themen, die Verwendung erzählerischer Kleingattungen, die scheinbar unverbundene Disposition der Erzähleinheiten, die Text-Bild-Kombinationen.

Allerdings möchte ich auch keiner vorschnellen Analogiebildung zwischen einem polyhistorischen Zeitschriftenprojekt des 17. Jahrhunderts und Kluges multimedialer Praxis zu Anfang des 21. Jahrhunderts Vorschub leisten. Die Kontrastierung kann aber wechselweise zu jeweils neuen Erkenntnissen führen: Für die Beschäftigung mit dem Werk Kluges würde das bedeuten, Bezugspunkte für sein ästhetisches Verfahren auch jenseits des Epochenraums seiner eigenen Selbstzuschreibungen zu suchen. Diese Vorgehensweise würde die in den letzten Jahren zu beobachtende Tendenz einer Historisierung von Kluges Werk stützen, also

${ }^{37}$ Vgl. Volker Meid, Die deutsche Literatur im Zeitalter des Barock. Vom Späthumanismus zur Frühaufklärung 1570-1740, München, Beck, 2009, S. 758.

38 Zit. nach Flemming Schock, Die Text-Kunstkammer. Populäre Wissenssammlungen des Barock am Beispiel der ,Relationes Curiosae‘ von E.W. Happel, Köln/Weimar/Wien, Böhlau, 2011, S. 129. Vgl. auch Uta Egenhoff, Berufsschriftstellertum und Journalismus in der Frühen Neuzeit. Eberhard Werner Happels Relationes curiosae im Medienverbund des 17. Jahrhunderts, Bremen, Edition Lumière, 2008, sowie Christian Meierhofer, Alles neu unter der Sonne. Das Sammelschrifttum der Frühen Neuzeit und die Entstehung der Nachricht, Würzburg, Königshausen \& Neumann, 2010.

39 Zit. nach Schock, Die Text-Kunstkammer, S. 134. 
einer verstärkten Einbindung und Kontextualisierung seiner Arbeit in literarische, mediale und theoriegeschichtliche Herleitungs- und Entstehungszusammenhänge. Auf der anderen Seite kann der Frühneuzeitforscher von dieser Kontrastierung ebenfalls profitieren: Für die erwähnte Zeitschrift Relationes Curiosae könnte das bedeuten, sie nicht in eine im Niedergang begriffene Endphase des Polyhistorismus einzuordnen, sondern ihre bemerkenswerte ästhetische Modernität in der Perspektive auf die Kluge'sche Poetik zu erweisen.

Zum Schluss möchte ich noch einmal in drei Stichpunkten den Stellenwert der Frühneuzeit-Epoche für das multimediale Werk Kluges zusammenfassen:

1. Die Epoche der Frühen Neuzeit dient Kluge zum einen als gewaltiges Stoffreservoir. Ihre historischen Ereignisse, Personen, Denkstile, Diskursfelder, Erzählmodelle und Erfahrungsgehalte werden quer durch das Werk als Vorlagen und Ausgangspunkte für die Geschichten, Interviews und Theoriereflexionen angeeignet.

2. Zum anderen hat die Epoche der Frühen Neuzeit eine Scharnierfunktion - sie verknüpft das Werk Kluges nach seiner Selbstauskunft sowohl mit dem kulturellen Erbe der Antike als auch mit der sozialphilosophischen Tradition der Kritischen Theorie. Zahlreiche Problemlagen, Konfliktlösungen und kulturelle Prozesse des Zeitalters sind für Kluge nach wie vor aktuell - die Frühmoderne ist eben auch eine Moderne, in der insbesondere der Humanismus und die Aufklärung zentrale Bezugspunkte sind.

3. Schließlich sind die Erzählmodelle und die wissensorganisatorischen Verfahrensweisen der Frühen Neuzeit für Kluges Werk relevant. So sind etwa die in den literarischen Arbeiten verwendeten Erzählelemente der Anekdote, der Kalendergeschichte oder des Dialogs Gattungen, die auf antike Traditionen zurückgehen, in der Frühen Neuzeit aber wiederbelebt und aktualisiert werden. Die Wissenspräsentation in polyhistorischer oder enzyklopädischer Form ist bei Kluge sowohl in den literarischen als auch in den Fernseharbeiten anzutreffen.

Die vorliegende Skizze hat nur einige wenige Fingerzeige darauf geben können, welche Dimensionen das Thema „Kluge und die Frühe Neuzeit“ hat. Sie ist in vielerlei Hinsicht erweiterbar, sowohl im Blick auf die Gegenstandsbereiche (insbesondere die Traditionsgeschichte der Text-Bild-Montagen ${ }^{40}$ ), als auch im Blick auf eine Vermehrung der Beispielanalysen. Hilfreich mag dabei die Fragestellung sein, die kürzlich in Bezug auf Kluges Verhältnis zur Prämoderne aufgeworfen wurde. Im Hinblick auf dessen Erzählweise verwendete Richard Faber die folgende, provozierend vereinfachende Formel: Der postmoderne Erzählkosmos Kluges entstünde durch die Verwendung prämoderner Erzählweisen wie etwa der Kalendergeschichte, kurz: „postmodern qua prämodern“41. Faber versah diese Formel aber mit einem, so könnte man sagen, sehr großen Fragezeichen. Da es sich immerhin lohnt, über diesen Zusammenhang weiter nachzudenken, sei neben das Fragezeichen im Rückblick auf die hier vorgestellten Überlegungen zumindest noch ein Ausrufezeichen gesetzt!

${ }^{40}$ Vgl. Pavel Novotný, Die Vorformen der literarischen Montage, Wuppertal, Arco-Verlag, 2012; Jörg Robert, Einführung in die Intermedialität, Darmstadt, Wiss. Buchgesellschaft, 2014.

${ }^{41}$ Faber, „Postmodern qua prämodern?“, S. 55. 
